\title{
ON THE EXISTENCE OF AD-NILPOTENT ELEMENTS
}

\author{
G. M. BENKART ${ }^{1}$ AND I. M. ISAACS ${ }^{2}$
}

\begin{abstract}
A condition sufficient to guarantee the nilpotence of a derivation of a Lie algebra is given. It is used to obtain an elementary proof that a finite dimensional Lie algebra over an algebraically closed field of arbitrary characteristic necessarily contains an ad-nilpotent element.
\end{abstract}

In recent papers [1], [2] and [3], ad-nilpotent elements have provided an effective means of characterizing certain simple Lie algebras in prime characteristic. An algebraic geometry argument can be used to prove the existence of ad-nilpotent elements in finite dimensional Lie algebras over algebraically closed fields; and in characteristic zero, existence also follows from the classical theory. In this paper we give an easy, elementary proof which works for algebraically closed fields of arbitrary characteristic. In fact, we prove slightly more.

If $L$ is a Lie algebra and $x \in L$, let $E(x)$ denote the span of the set of eigenvectors of ad $x$ in $L$. If $y, z \in L$ are eigenvectors of $\operatorname{ad} x$, then $[y z]$ is either zero or is itself an eigenvector. It follows that $E(x)$ is a subalgebra of $L$.

LEMma. Let $L$ be a finite dimensional Lie algebra over an algebraically closed field $F$ and let $D$ be a derivation of L. Suppose $x \in L$ and that $D^{n}(E(x))=0$ for some integer $n$. Then $D$ is nilpotent on $L$.

Proof. For $\lambda \in F$, let $L_{\lambda}$ denote the maximal subspace of $L$ on which $D-\lambda$ is nilpotent. As is well known, $\left[L_{\lambda} L_{\mu}\right] \subseteq L_{\lambda+\mu}$. Now $x \in E(x) \subseteq L_{0}$ and so $\left[x L_{\lambda}\right] \subseteq L_{\lambda}$ for all $\lambda$. If $L_{\lambda} \neq 0$, then ad $x$ has an eigenvector in $L_{\lambda}$ and so $0 \neq L_{\lambda} \cap E(x) \subseteq L_{\lambda} \cap L_{0}$ and thus $\lambda=0$. Since $L=\Sigma_{\mu} L_{\mu}$, we have $L=L_{0}$.

We now prove our theorem. It is slightly stronger than the assertion in the abstract which is obtained from it by taking $X$ to be $L-\{0\}$. The option of taking smaller sets $X$, however, is occasionally useful [2].

TheOREM. Let $L$ be a finite dimensional Lie algebra over $F$ where $F$ is algebraically closed. Let $X \subseteq L$ be a nonempty subset such that for every $x \in X$, all eigenvectors of ad $x$ lie in $X$. Then ad $y$ is nilpotent for some $y \in X$.

Received by the editors September 28, 1976.

AMS (MOS) subject classifications (1970). Primary 17B40.

Key words and phrases. Ad-nilpotent element, modular Lie algebra.

${ }^{1}$ Research supported by NSF Grant MCS 73-08615 A03.

${ }^{2}$ Research supported by NSF Grant MCS 74-06398 A02. 
Proof. Use induction on $\operatorname{dim} L$. Let $x \in X$. Suppose $E(x)<L$. Now $E(x) \cap X$ is nonempty (since it contains $x$ ) and satisfies the hypotheses of the theorem in the algebra $E(x)$. By the inductive hypothesis, there exists $y \in E(x) \cap X$ such that ad $y$ is nilpotent on $E(x)$. By the Lemma, ad $y$ is nilpotent on $L$.

We may suppose then that $E(x)=L$ for all $x \in X$. Let $x \in X$. If ad $x$ is not nilpotent, it has some eigenvector $y$ with $\left[\begin{array}{ll}x & y\end{array}\right]=\lambda y$ and $\lambda \neq 0$. Then $(\operatorname{ad} y)^{2}(x)=0$. However, $y \in X$ and so $E(y)=L$ and ad $y$ is semisimple on $L$. Thus $(\operatorname{ad} y)(x)=0$. This contradicts $[y x]=-\lambda y \neq 0$ and proves the result.

\section{REFERENCES}

1. G. M. Benkart, On inner ideals and ad-nilpotent elements, Trans. Amer. Math. Soc. (to appear).

2. G. M. Benkart, I. M. Isaacs and J. M. Osborn, Lie algebras with self-centralizing ad-nilpotent elements (to appear).

3. H. Strade, Nonclassical simple Lie algebras and strong degeneration, Arch. Math. (Basel) 24 (1973), 482-485. MR 51 \# 12963.

Department of Mathematics, University of Wisconsin, Madison, Wisconsin 53706 